This item was submitted to Loughborough's Research Repository by the author.

Items in Figshare are protected by copyright, with all rights reserved, unless otherwise indicated.

\title{
The two-parameter soliton family for the interaction of a fundamental and its
} second harmonic

PLEASE CITE THE PUBLISHED VERSION

LICENCE

CC BY-NC-ND 4.0

REPOSITORY RECORD

Grimshaw, Roger H.J., E.A. Kuznetsov, and E.G. Shapiro. 2019. "The Two-parameter Soliton Family for the Interaction of a Fundamental and Its Second Harmonic". figshare. https://hdl.handle.net/2134/766. 


\title{
The two-parameter soliton family for the interaction of a fundamental
} and its second harmonic

\author{
R.H.J. Grimshaw ${ }^{(a)}$, E. A. Kuznetsov ${ }^{(b)}$, and E.G. Shapiro ${ }^{(c)}$ \\ (a) - Department of Mathematics and Statistics, Monash University, Clayton, Vic 3800, Australia \\ (b) - Landau Institute for Theoretical Physics, 2 Kosygin str., 117334 Moscow, Russia \\ (c) - Institute of Automation and Electrometry, 630090 Novosibirsk, Russia
}

(October 30, 2000)

\begin{abstract}
For a system of interacting fundamental and second harmonics the soliton family is characterized by two independent parameters, a soliton potential and a soliton velocity. It is shown that this system, in the general situation, is not Galilean invariant. As a result, the family of movable solitons cannot be obtained from the rest soliton solution by applying the corresponding Galilean transformation. The region of soliton parameters is found analytically and confirmed by numerical integration of the steady equations. On the boundary of the region the solitons bifurcate. For this system there exist two kinds of bifurcation: supercritical and subcritical. In the first case the soliton amplitudes vanish smoothly as the boundary is approached. Near the bifurcation point the soliton form is universal, determined from the nonlinear Schrodinger equation. For the second type of bifurcations the wave amplitudes remain finite at the boundary. In this case the Manley-Rowe integral increases indefinitely as the boundary is approached, and therefore according to the VK-type stability criterion, the solitons are unstable.
\end{abstract}

\section{INTRODUCTION}

It is well-known that three wave packets which carrier frequencies satisfying the triad resonant condition can form bound states - solitons - due to their mutual nonlinear interaction (see e.g. [1]). The corresponding equations are called the three-wave system [2]. The three-wave system describes spatial solitons as well as spatial-temporal solitons in $\chi^{(2)}$ media $[1,3]$. This system couples amplitudes of three quasi-monochromatic waves due to quadratic nonlinearity. A special case of such an interaction is that of the first (fundamental) and second harmonic, given by

$$
2 \omega(k)=\omega(2 k)
$$

where $\omega=\omega(k)$ is the dispersion law and $k$ is the wave vector. In this case the three-wave system transforms into a pair of equations for the amplitudes of the first and second harmonics. When the difference in group velocities between these first and second harmonics is small enough (which is typical for nonlinear optics) it is necessary to take into account wave dispersion. In this case this model can be considered as a vector nonlinear Schrodinger system but with quadratic nonlinearity. The balance between nonlinear interaction and dispersion results in the existence of solitons. If the difference in group velocities is large enough, then each wave packet propagates away with its group velocity, and the system cannot form a bound state between the first and second harmonic. Moreover, in this case this system is close to being integrable. For zero dispersion it can be integrated by means of the inverse scattering transform $[4,5]$.

The system of a fundamental frequency (FF) interacting with its second harmonic (SH) has also another very interesting application to solitary waves propagating with a constant velocity in a nonlinear medium without change of shape. Such objects have been called solitons [6]. These objects are really stationary, unlike, for instance, spatial-temporal solitons in a $\chi^{2}$ medium where only the envelopes of the amplitudes can be considered stationary. To distinguish these solitons 
from really stationary solitons, it was suggested in [6] to call the former quasi-solitons. For instance, breathers described by the Sine-Gordon equation will be an example of such quasi-solitons.

It was shown in [6] and [9] that when the soliton velocity $v$ is close to the minimum phase velocity of linear waves, $v_{c r}=\min (\omega(k) / k)$, the Fourier spectrum of the stationary pulse represents a set of peaks. Their positions in the frequency domain correspond to the frequency related to the minimal phase velocity (fundamental harmonic) and to its multiple harmonics. The width of these peaks vanishes when the critical velocity $v_{c r}$ is approached. This allows us to introduce an envelope for each peak, and apply a standard multi-scale expansion. In the case when the carrier frequency, corresponding to the critical velocity does not satisfy the resonance condition (1), then the envelope of the fundamental harmonic obeys the nonlinear Schroedinger equation (NLS). This phenomenon was first discovered for gravity-capillary waves $[7,8]$. Later it was understood that this is the general situation for an arbitrary soliton if its amplitude vanishes at $v=v_{c r}$ (i.e. does not have a jump at the critical velocity). If the carrier frequency for the fundamental harmonic is close to the resonance condition (1) then the corresponding equations for a steady pulse transform into the steady (timeindependent) equations for the interacting fundamental and second harmonics. The stability of these soliton solutions with respect to modulation perturbations can be described in the framework of the unsteady system for fundamental and second harmonics.

When the difference in group velocities for FF and $\mathrm{SH}$ is small enough, then it is necessary to take into account dispersion (plus diffraction in the multi-dimensional case), and then the system of interacting fundamental and second harmonics contains three free parameters: the phase mismatch $\Omega$, which characterizes how far the carrier frequencies of the first and second harmonics are from resonance, and two dispersion coefficients $\omega_{1}^{\prime \prime}$ and $\omega_{2}^{\prime \prime}$ where 1,2 stand for FF and SH respectively, and prime means derivative with respect to $k_{1,2}$ where $k_{2}=2 k_{1}$. In the multi-dimensional case, instead of dispersion coefficients two dispersion tensors arise. All other parameters can be excluded by simple rescaling. The behavior of solitons depends significantly on these three parameters. In the simplest variant soliton solutions are determined by one internal parameter $\lambda^{2}$, which can be considered as a "chemical potential". Until now, mainly such solutions have been treated. We would like to pay attention to the fact that the system of interacting fundamental and second harmonics (as well as the three-wave system) is not Galilean invariant in the general situation. In particular, this means that the system must have a more broad soliton family than could be considered before. The velocity of the soliton, together with the "chemical potential" are two inner independent parameters of the soliton family. In the present paper we study this two-parameter family both analytically and numerically. We show that these two parameters are not arbitrary indeed: there are some restrictions imposed on them which follow from the conditions for soliton existence. This yields a two-dimensional region in the parameter space. Passing across the boundary of this region, a soliton undergoes bifurcations. In this paper we show that for this system two types of bifurcation are possible. The first is a supercritical bifurcation, when the first harmonic amplitude $\psi_{1}$ for the soliton solution vanishes smoothly while the second harmonic amplitude depends on $\psi_{1}$ quadratically. Near such a bifurcation a soliton solution transforms into the soliton of the nonlinear Schrodinger equation (NLS) that is embedded in the general scheme suggested in [6], [9]. As we show in this paper such solutions are stable for the one-dimensional case.

Another possibility is a subcritical bifurcation which takes place when the characteristic size for the second harmonic becomes infinite as the soliton parameters approach the boundary. In this case near the boundary the amplitude of the second harmonic remains finite, but the amplitude $\psi_{1}$ vanishes. Correspondingly, close to the boundary the Manley-Rowe integral in this case becomes infinite. The derivative of this integral relative to the parameter $\lambda^{2}$ becomes negative, so that, in accordance with the Vakhitov-Kolokolov (VK) type of criteria [10,11], there is soliton instability. In this case, however, this criterion is only a sufficient criterion for instability : it cannot be used to establish stability. The original VK stability criterion was derived first for the NLS equation [10]:

$$
\partial N / \partial \lambda^{2}>0
$$


Here $N$ is the number of particles in the soliton solution and $\varepsilon=-\lambda^{2}<0$ is the energy of the NLS soliton in the bound state. For the NLS this criterion is simultaneously necessary and sufficient. For a positive derivative, i.e., when the addition of a single particle decreases the energy $\varepsilon$, the soliton is stable. In the opposite case, where a level is expelled as $N$ increases, the soliton is unstable (in this case the derivative in (2) is negative). The difference in the stability criteria between the NLS and the FF-SH system (being a two-component NLS system), is connected with the vector character of the latter (for details see [11]). Just for this reason, it is impossible to generalize the criterion (2) to the FF-SH system completely.

\section{BASIC EQUATIONS}

The equations of motion describing the interaction of the first (fundamental) and second harmonics can be written as follows:

$$
\begin{aligned}
i \frac{\partial \psi_{1}}{\partial t}+\frac{1}{2} \omega_{1}^{\prime \prime} \psi_{1 x x} & =-2 \psi_{2} \psi_{1}^{*}, \\
i \frac{\partial \psi_{2}}{\partial t}-\Omega \psi_{2}+\frac{1}{2} \omega_{2}^{\prime \prime} \psi_{2 x x} & =-\psi_{1}^{2}
\end{aligned}
$$

where $\psi_{1}$ and $\psi_{2}$ are amplitudes for the first and second harmonics respectively, and the parameter $\Omega$ in (3) characterizes the phase mismatch. Here, for simplicity, we consider only the one-dimensional case. The corresponding generalization to the multi-dimensional case is straightforward. In particular, in the multi-dimensional case one needs to change the 1 D operators $\omega_{l}^{\prime \prime} \partial_{x}^{2}(l=1,2)$ in $(3,4)$ to

$$
\frac{\partial^{2} \omega_{l}\left(k_{l}\right)}{\partial k_{l i} \partial k_{l j}} \frac{\partial^{2}}{\partial x_{i} \partial x j} .
$$

The system under consideration $(3,4)$ is Hamiltonian:

$$
i \frac{\partial \psi_{l}}{\partial t}=\frac{\delta H}{\delta \psi_{l}^{*}}
$$

with the Hamiltonian

$$
H=\int \Omega\left|\psi_{2}\right|^{2} d x+\sum_{l} \int \frac{1}{2} \omega_{l}^{\prime \prime}\left|\psi_{l x}\right|^{2} d x-\int\left(\psi_{1}^{* 2} \psi_{2}+\text { c.c. }\right) d x .
$$

Besides the Hamiltonian, this system conserves also the Manley-Rowe integral

$$
N=\int\left(\left|\psi_{1}\right|^{2}+2\left|\psi_{2}\right|^{2}\right) d x
$$

which is a consequence of gauge invariance of the system. This integral has also be regarded as an adiabatic invariant which appears as a result of average of the original system over fast oscillations corresponding to the carrier frequencies of two resonant wave packets. From the definition (7), the Manley-Rowe integral is a positive quantity.

Another integral of motion for Eqs. $(3,4)$ is the momentum,

$$
P=-\frac{i}{2} \sum_{l} \int\left(\psi_{l}^{*} \psi_{l x}-\psi_{l x}^{*} \psi_{l}\right) d x
$$

which is a consequence of the invariance of the system to spatial translations. The latter, however, does not guarantee that the equations of motion will be Galilean invariant. To check this, let us perform two transformations. The first is passing to the coordinate system moving with velocity $v$, 


$$
x^{\prime}=v-v t, t^{\prime}=t,
$$

and another is a simple gauge transform:

$$
\psi_{1} \rightarrow \psi_{1} e^{-i \omega t+i k x}, \quad \psi_{2} \rightarrow \psi_{2} e^{-i 2 \omega t+i 2 k x} .
$$

Then we require that the obtained equations have a similar form to the original system. Simple calculations show that this demand can be satisfied if and only if

$$
2 p_{1}=p_{2}
$$

where $p_{l}=1 / \omega_{l}^{\prime \prime}$. Under this condition only, we have Galilean invariance. In all other cases the system $(3,4)$ is not Galilean invariant. The same situation occurs for the three-wave system which describes the interaction of three resonant wave packets (compare with [11]). For the three-wave system the analog of $(10)$ is written as follows

$$
p_{1}+p_{2}=p_{3} .
$$

The absence of Galilean invariance in the general case for the system $(3,4)$ means that movable soliton solutions cannot be transformed by means of the transformations $(8,9)$ to the rest soliton. In other words, the soliton velocity $v$ itself is a new independent parameter, which together with the energy of solitons (as bound states) $\varepsilon=-\lambda^{2}$ defines a two-parameter soliton family. This family is given as follows:

$$
\psi_{1}(x, t)=\psi_{1 s}(x-v t) e^{i \lambda^{2} t}, \quad \psi_{2}(x, t)=\psi_{2 s}(x-v t) e^{2 i \lambda^{2} t},
$$

where the amplitudes $\psi_{1 s}$ and $\psi_{2 s}$ satisfy the equations:

$$
\begin{aligned}
-\lambda^{2} \psi_{1}-i v \partial_{x} \psi_{1}+\frac{1}{2} \omega_{1}^{\prime \prime} \partial_{x}^{2} \psi_{1} & =-2 \psi_{2} \psi_{1}^{*}, \\
-2 \lambda^{2} \psi_{2}-i v \partial_{x} \psi_{2}-\Omega \psi_{2}+\frac{1}{2} \omega_{2}^{\prime \prime} \partial_{x}^{2} \psi_{2} & =-\psi_{1}^{2} .
\end{aligned}
$$

Here and below the index $s$ for $\psi_{1 s}$ and $\psi_{2 s}$ is temporarily omitted.

It is easy to verify that a solution of the stationary equations is a stationary point of the Hamiltonian $H$ for fixed $N$ and momentum $P$ : equations $(11,12)$ follow from the variational problem

$$
\delta\left(H+\lambda^{2} N-v P\right)=0 .
$$

In order to have localized solution of the stationary system it is necessary to require that two operators

$$
\begin{aligned}
& A_{1}=-\lambda^{2}-i v \partial_{x}+\frac{1}{2} \omega_{1}^{\prime \prime} \partial_{x}^{2}, \\
& A_{2}=-2 \lambda^{2}-i v \partial_{x}-\Omega+\frac{1}{2} \omega_{2}^{\prime \prime} \partial_{x}^{2}
\end{aligned}
$$

must be sign (negative or positive) definite to provide exponentially decreasing behaviour at infinity. Physically, this requirement means absence of Cherenkov radiation by solitons. Indeed, the condition on the signature of the operators is more restrictive: the operators $A_{1}$ and $A_{2}$ must be simultaneously negative (or positive) definite. This follows from the two integral relations which can be obtained from the variational principle (13) and directly from the stationary equations $(11,12)$ after multiplying the first equation by $\psi_{1}^{*}$, the second one by $2 \psi_{2}^{*}$, with summation of the obtained results, followed by their integration over $x$ (for details, see [11]).

In the case when the operators are negative definite, the following conditions must be fulfilled: 


$$
\begin{aligned}
\omega_{1,2}^{\prime \prime} & >0, \\
-\lambda^{2}+\frac{1}{2} \frac{v^{2}}{\omega_{1}^{\prime \prime}} & <0, \\
-2 \lambda^{2}-\Omega+\frac{1}{2} \frac{v^{2}}{\omega_{2}^{\prime \prime}} & <0 .
\end{aligned}
$$

These requirements guarantee absence of Cherenkov radiation by stationary propagating solitons (for details, see papers $[6,9]$ ).

The conditions (14-16) define the region of parameters where soliton solutions are possible. To find this region it is convenient to introduce instead of $\lambda^{2}$ a new quantity $\Lambda^{2}=\lambda^{2}-p_{1} v^{2} / 2$ which in accordance with (15) has to be a positive quantity. As a result, the last inequality (16) reads as

$$
2 \Lambda^{2}+\left(2 p_{1}-p_{2}\right) \frac{v^{2}}{2}+\Omega>0
$$

Depending on the signs of $\kappa=2 p_{1}-p_{2}$ and $\Omega$ we have four possibilities:

1. $\kappa>0, \Omega>0$. In this case the inequality (17) is satisfied automatically. The allowed region for the soliton parameters is the quarter-plane $\Lambda_{1}^{2}>0, v^{2} \geq 0$.

2. $\kappa>0, \Omega<0$. The allowed region for the soliton parameters is the quarter-plane $\Lambda^{2}>0$ and $v^{2} \geq 0$ except the triangular region near origin bounded by the straight line $2 \Lambda^{2}+\kappa v^{2} / 2=|\Omega|$.

3. $\kappa<0, \Omega>0$. The allowed region for the parameters is the quarter-plane $\Lambda^{2}>0$ and $v^{2} \geq 0$ except the region below the straight line $2 \Lambda^{2}=|\kappa| v^{2} / 2-\Omega$.

4. $\kappa<0, \Omega<0$. The allowed region is the quarter-plane $\Lambda^{2}>0$ and $v^{2} \geq 0$ except the region below the straight line $2 \Lambda^{2}=|\kappa| v^{2} / 2+|\Omega|$. In this case $\Lambda^{2}$ can not reach zero value.

It is interesting to note that $\kappa=0$ recovers Galilean invariance of the equations (3), (4). In this case both criteria (14) and (17) when expressed through $\Lambda^{2}$ do not contain the velocity:

$$
\Lambda^{2}>0,2 \Lambda^{2}+\Omega>0
$$

\section{SOLITONS AND BIFURCATIONS}

Next we shall analyze the soliton solutions which are defined from the system (11), (12). By the transformation,

$$
\psi_{l} \rightarrow \psi_{1} e^{i p_{1} v x}, \psi_{2} \rightarrow \psi_{2} e^{2 i p_{1} v x},
$$

and rescaling the amplitudes $\psi_{l}$, this system can be rewritten as follows:

$$
\begin{aligned}
-\Lambda_{1}^{2} \psi_{1}+\partial_{x}^{2} \psi_{1} & =-2 \psi_{2} \psi_{1}^{*}, \\
-\Lambda_{2}^{2} \psi_{2}+\left(\partial_{x}-i k\right)^{2} \psi_{2} & =-\psi_{1}^{2}
\end{aligned}
$$

where $k=\kappa v$ and

$$
\Lambda_{1}^{2}=2 p_{1} \Lambda^{2}, \quad \Lambda_{2}^{2}=2 p_{2}\left(2 \Lambda^{2}+\kappa v^{2} / 2+\Omega\right) .
$$

The ordinary differential equations (18) and (19) have two integrals. The first is obtained by multiplying Eq. (18) by $\psi_{1}^{*}$, subtracting the complex conjugate, and then repeating the operation for Eq. (19). Then multiplying the latter result by 2, and adding to the former, we get: 


$$
\psi_{1}^{*} \psi_{1 x}-\psi_{1 x}^{*} \psi_{1}+2\left(\psi_{2}^{*} \psi_{2 x}-\psi_{2 x}^{*} \psi_{2}\right)-4 i k\left|\psi_{2}\right|^{2}=M
$$

which has a meaning of "angular momentum" for the system (18, 19). Another integral, "energy", can be obtained in a similar way. The only difference is that it is first necessary to multiply Eq.(18) by $\psi_{1 x}^{*}$ and then add the complex conjugate. The result is:

$$
\left|\psi_{1 x}\right|^{2}+\left|\psi_{2 x}\right|^{2}-\Lambda_{1}^{2}\left|\psi_{1}\right|^{2}-\left(\Lambda_{2}^{2}+k^{2}\right)\left|\psi_{2}\right|^{2}+\psi_{2}^{*} \psi_{1}^{2}+\psi_{2} \psi_{1}^{* 2}=E .
$$

For a soliton solution $\left(\psi_{1,2} \rightarrow 0\right.$ as $\left.|x| \rightarrow \infty\right)$ both integrals are equal to zero. Here we have two cases $k=0$ and $k \neq 0$. In the first case a soliton solution can be taken purely real and so the first integral becomes equal to zero identically. Thus, in this case only the "energy" integral survives, which can be used to reduce the order of the system of ordinary differential equations. In the second case the solution remains complex.

It is worth noting that the conditions (14)-(16) for localized solutions of the system (18), (19) correspond simply to the positivity of $\Lambda_{1}^{2}$ and $\Lambda_{2}^{2}$. Thus, the boundary of the soliton parameter region is given by the two equations $\Lambda_{1}^{2}=0$ (when $\Lambda_{2}^{2}>0$ ) and $\Lambda_{2}^{2}=0$ (when $\Lambda_{1}^{2}>0$ ). Accordingly, we have two variants of soliton degeneracy.

\section{A. Supercritical bifurcations}

Consider first how the soliton family looks like near the parameter boundary $\Lambda_{1}^{2}=0$ (when $\Lambda_{2}^{2}>0$ ) First, note that in Eq. (18) $\psi_{1}$ near this boundary changes on the scale $l \sim 1 / \Lambda_{1}$. In this limit the wave function $\psi_{2}$ has the same characteristic scale, i.e, $l \sim 1 / \Lambda_{1}$. Therefore in Eq. (19) we can neglect the derivatives and, as a result, obtain a local relation between $\psi_{1}$ and $\psi_{2}$ :

$$
\left(\Lambda_{2}^{2}+k^{2}\right) \psi_{2}=\psi_{1}^{2}
$$

Substitution of this expression into (18) leads to the stationary nonlinear Schrodinger equation (NLS):

$$
-\Lambda_{1}^{2} \psi_{1}+\partial_{x}^{2} \psi_{1}+\frac{2}{\Lambda_{2}^{2}+k^{2}}\left|\psi_{1}\right|^{2} \psi_{1}=0
$$

Its solution is the NLS soliton:

$$
\psi_{1}=\frac{\Lambda_{1}}{\sqrt{\Lambda_{2}^{2}+k^{2}}} \operatorname{sech}\left(\Lambda_{1} x\right) .
$$

In this case the second harmonic amplitude is given by the expression

$$
\psi_{2}=\frac{\Lambda_{1}^{2}}{\left(\Lambda_{2}^{2}+k^{2}\right)^{2}} \operatorname{sech}^{2}\left(\Lambda_{1} x\right) .
$$

In this asymptotic regime, the main contribution in the Manley-Rowe integral is given by the first harmonic (23):

$$
N \approx \frac{2 \Lambda_{1}}{\Lambda_{2}^{2}+k^{2}}
$$

In Fig. 1 we show the dependence of the Manley-Rowe integral $N$ on $\Lambda_{1}$ for the case $k=0$ and $\Lambda_{2}^{2}=1$, obtained by numerical integration of the system (18),(19). For small $\Lambda_{1} N$ changes linearly in accordance with the analytical dependence (24). For larger $\Lambda_{1}$ we have a positive deviation from this linear dependence. In all our numerical work we also checked that for $\Lambda_{1}^{2}<0$, as well as for 
$\Lambda_{1}^{2}<0$, soliton solutions are absent, which is in complete agreement with the definition of the soliton region given by $(14-16)$.

Thus, while approaching the boundary $\Lambda_{1}^{2}=0$ the first harmonic amplitude undergoes a supercritical bifurcation: $\max \left|\psi_{1}\right| \sim \Lambda_{1}$ and $\psi_{2}$ vanishes like $\Lambda_{1}^{2}$. For $\Lambda_{1}^{2}<0$ Eq. (22) has no localized solution at all. Note that this case is completely embedded in the general situation for this type of bifurcations of solitons (cf. [6], [9]).

To conclude this subsection, we would like to point out that the reduction of the FF-SH system to the NLS was first obtained in the paper [1] at the case when the phase mismatch parameter is large enough. Later it was discussed in many other papers (see, for instance, the recent paper [14] and the review [15]).

\section{B. Subcritical bifurcations}

Now let us consider how solitons behave near the other boundary $\Lambda_{2}^{2}=0$ (when $\Lambda_{1}^{2}$ and $k$ are not equal to zero). First we shall analyze the special case $k=0$ when the system (18), (19) takes the form:

$$
\begin{aligned}
& -\Lambda_{1}^{2} \psi_{1}+\partial_{x}^{2} \psi_{1}=-2 \psi_{2} \psi_{1} \\
& -\Lambda_{2}^{2} \psi_{2}+\partial_{x}^{2} \psi_{2}=-\psi_{1}^{2}
\end{aligned}
$$

Here without lose of generality we put $\psi_{1}=\psi_{1}^{*}$ so that Eq.(25) transforms into the stationary Schrodinger equation for $\psi_{1}$ and $U(x)=2 \psi_{2}(x)$ there serves as a potential. The latter quantity is found from the second equation (26) by means of a Green's function:

$$
\psi_{2}=\frac{1}{2 \Lambda_{2}} \int_{-\infty}^{\infty} e^{-\Lambda_{2}\left|x-x^{\prime}\right|} \psi_{1}^{2}\left(x^{\prime}\right) d x^{\prime} .
$$

Thus one can see that $\psi_{2}(x)$ decreases exponentially for large $x$ and the small parameter $\Lambda_{2}$ defines the largest scale $L=\Lambda_{2}^{-1}$ in this problem:

$$
\psi_{2} \approx \frac{e^{-\Lambda_{2}|x|}}{2 \Lambda_{2}} \int_{-\infty}^{\infty} \psi_{1}^{2}\left(x^{\prime}\right) d x^{\prime} \text { for }|x| \sim L .
$$

On the other hand, in the stationary Schrodinger equation (25) the value $\Lambda_{1}^{2}$ can be considered as the energy of the bound state and will yield the smallest scale $l=\Lambda_{1}^{-1} \ll L$ in this problem. Further, as we show below, the characteristic scale $a$ of $\psi_{1}(x)$ lies between $l$ and $L$ :

$$
L \gg a \gg l .
$$

This allows one to neglect the second derivative term in (25) and so estimate the maximum value of $\psi_{2}$ (attained at $x=0$ ):

$$
\max \left(\psi_{2}\right) \approx \Lambda_{1}^{2} / 2 .
$$

Comparing (28) and (30) we arrive at the following estimate for the integral

$$
\int_{-\infty}^{\infty} \psi_{1}^{2} d x \approx \Lambda_{1}^{2} \Lambda_{2}
$$

Thus, this integral vanishes as $\Lambda_{2} \rightarrow 0$. At the same time the integral of $\psi_{2}^{2}$ becomes infinitely large: the maximum value remains constant and the characteristic scale becomes infinite as $\Lambda_{2}$ tends to zero. By this argument, the corresponding Manley-Rowe integral will diverge as 


$$
N \approx 2 \int \psi_{2}^{2} d x \approx \frac{\Lambda_{1}^{4}}{2 \Lambda_{2}} .
$$

The main contribution to this integral is given by the second harmonic, and correspondingly the contribution from the first harmonic is small.

Let us next consider the behavior of $\psi_{1}(x)$. To estimate its amplitude and to find its characteristic size we shall assume that

$$
\psi_{1}(x)=A \phi_{1}(\xi), \quad \text { where } \quad \xi=\alpha x \quad \text { and } \quad \alpha=\frac{1}{a} .
$$

Substituting (32) into the integral (27) and taking into account the relations between the scales (29) we find that

$$
\begin{gathered}
\psi_{2}=\frac{A^{2} C_{0}}{2 \alpha \Lambda_{2}} \exp \left(-\Lambda_{2}|x|\right)+\frac{A^{2}}{\alpha^{2}} \phi_{2}(\xi), \\
\text { where } \quad C_{0}=\int_{-\infty}^{\infty} \phi_{1}^{2}(\xi) d \xi \quad \text { and } \quad \phi_{2 \xi \xi} \approx-\phi_{1}^{2} .
\end{gathered}
$$

For large $|x|(\sim L)$, this expression has the same asymptotic behaviour as (28). For small $|x| \sim a$ the first term can be expanded so that the potential $\left(U=2 \psi_{2}\right)$ can be represented as follows

$$
\begin{gathered}
U(x)=U_{0}+\frac{A^{2}}{\alpha^{2}} V(\xi) \\
\text { where } \quad U_{0}=\frac{A^{2} C_{0}}{\alpha \Lambda_{2}}, \quad \text { and } \quad V(\xi)=\left[-|\xi|+2 \phi_{2}(\xi)\right] .
\end{gathered}
$$

Here $U_{0}$ gives the constant background and the potential $V(\xi)\left(\ll U_{0}\right)$ provides the bound state for $\psi_{1}$. In this case $U_{0}$ is approximately equal to $\Lambda_{1}^{2}$ which coincides with (30) for the maximum value of $\psi_{2}$. The small difference between $\Lambda_{1}^{2}$ and $2 \max \left(\psi_{2}\right)$ is just $\alpha^{2}$, so that:

$$
\Lambda_{1}^{2}=\frac{A^{2} C_{0}}{\alpha \Lambda_{2}}+\alpha^{2}, \quad \text { and } \quad A^{2}=\alpha^{4} .
$$

As a result, for these scales equation (25) takes the form:

$$
-\phi_{1}+\partial_{\xi}^{2} \phi_{1}+V(\xi) \phi_{1}=0 .
$$

We now see that the scaling of the small parameters yields the relations:

$$
\frac{A^{2} C_{0}}{\alpha} \approx \Lambda_{1}^{2} \Lambda_{2}
$$

from which it follows that:

$$
C_{0} \alpha^{3} \approx \Lambda_{1}^{2} \Lambda_{2},
$$

and the amplitude of $\psi_{1}$ is of the order:

$$
A \approx \frac{\Lambda_{1}^{4 / 3} \Lambda_{2}^{2 / 3}}{C_{0}^{2 / 3}}
$$


Thus, we have shown that for the case $k=0$ the amplitude of the second harmonic (SH) remains constant at the bifurcation point, but its size becomes infinitely large. In contrast to SH, the FF amplitude vanishes as $\Lambda_{2}^{2 / 3}$, but its size grows as $\Lambda_{2}^{-1 / 3}$. This means that we have in this case a subcritical bifurcation.

In spite of this behavior for $\psi_{1}$, the main contribution to the Manley-Rowe integral comes from the SH, since the input from the FF is small. This statement holds also at $k \neq 0$. To establish this fact it is not necessary to know the solution for $\psi_{1}$, it is enough to estimate contributions from the FF and SH in the Manley-Rowe integral.

Consider the stationary equations (18) and (19) for $k \neq 0$. In this case first it is convenient to exclude $k$ from the second equation (19) by means of the transform:

$$
\begin{aligned}
& \psi_{2} \rightarrow \psi_{2} e^{i k x}, \\
& \psi_{1} \rightarrow \psi_{1} e^{i k x / 2} .
\end{aligned}
$$

Then Eqs. (18), (19) become:

$$
\begin{aligned}
-\Lambda_{1}^{2} \psi_{1}+ & \left(\partial_{x}+i k / 2\right)^{2} \psi_{1}=-2 \psi_{2} \psi_{1}^{*}, \\
-\Lambda_{2}^{2} \psi_{2}+\partial_{x}^{2} \psi_{2} & =-\psi_{1}^{2}
\end{aligned}
$$

so that the second equation has the same form when $k=0$, and hence $\psi_{2}$ is given by the same expression (27).

Assuming that the amplitude $\psi_{1}$ has a characteristic scale larger than $\Lambda_{1}^{-1}$ and $k^{-1}$, one can get an estimate for $\psi_{2}$ at $x=0$ (analogous to $(30)$ ):

$$
\max \left|\psi_{2}\right| \simeq\left(\Lambda_{1}^{2}+k^{2} / 4\right) / 2
$$

so that it remains finite at $\Lambda_{2}=0$. As in the case $k=0$, the characteristic size of $\left|\psi_{2}\right|$ grows like $\Lambda_{2}^{-1}$. From this point of view, the type of bifurcation remains the same as it was at $k=0$ : this is the subcritical bifurcation. As far as $\psi_{1}$ is concerned, its contribution to the Manley-Rowe integral becomes infinitely small with respect to that from $\mathrm{SH}$ :

$$
\int\left|\psi_{1}\right| d x \sim \Lambda_{2}
$$

This follows from comparison of Eq. (28) at $x=0$ and Eq. (38). The latter indicates that the FF amplitude must vanish at $\Lambda_{2}=0$.

Thus, for both cases $\left(k=0\right.$ and $k \neq 0$ ) the Manley-Rowe integral diverges like $1 / \Lambda_{2}$ for small $\Lambda_{2}$ :

$$
N \approx 2 \int\left|\psi_{2}\right|^{2} d x \sim 1 / \Lambda_{2}
$$

Thus, the function $N\left(\Lambda_{2}\right)$ has a negative derivative near $\Lambda_{2}=0$, which, due to the VK-type criterion [11], corresponds to instability of solitons. At $k=0$ it is possible to prove. For $k \neq 0$ it is an open problem, but it looks very reasonable to expect instability also in this case because the instability holds for small $k$.

In Fig. 2 we show the dependence of $N$ on $\Lambda_{2}$ for the case $\Lambda_{1}^{2}=\Lambda_{2}^{2}+1$ and $k=0$, obtained from our numerical solutions. It was found that such behavior of $N$ with respect to $\Lambda_{2}$ is typical for all cases including $k=0$ : for small values of $\Lambda_{2} N$ diverges, for intermediate values it has a minimum at $\Lambda_{2}=\Lambda_{2 \min }$ and grows for larger $\Lambda_{2}$. When $N \rightarrow \infty$ as $\Lambda_{2} \rightarrow 0$, the dependence $N\left(\Lambda_{2}\right)$ can be approximated by the function

$$
f\left(\Lambda_{2}\right)=\frac{a}{\Lambda_{2}}+b
$$


where for $\Lambda_{1}^{2}=1+\Lambda_{2}^{2}$ and $k=0, a=0.537, b=3.33$. So we can see a nice correspondence with theoretical formula (31) where $a=1 / 2$.

In Fig. 3 we show the dependence of $N\left(\Lambda_{2}\right)$ for this case, where the dashed line corresponds to the approximation (39). The same dependence $N\left(\Lambda_{2}\right)$ is displayed on Fig.4 for $k=1$ with the same value of $\Lambda_{1}=1$ (in this case $a=0.763$ and $b=4.299$ ). As $\Lambda_{2} \rightarrow 0$ our numerics demonstrate that the maximum amplitude of the second harmonic tends to a constant (Fig.5) but its width grows (Fig.6) which causes the divergence of $N$ for small $\Lambda_{2}$. Simultaneously the first harmonic amplitude vanishes and its width increases slightly as $\Lambda_{2}$ approaches zero (see also Fig.7). ${ }^{1}$. As a result, the contribution of the first harmonic to $N$ becomes small compared with that from the second harmonic.

Thus, the function $N\left(\Lambda_{2}\right)$ for the soliton solution has a negative derivative in the band $0<\Lambda_{2}<$ $\Lambda_{2 \min }$ where, according to the VK-type criterion [11], the considered soliton solutions should be expected to be unstable against small perturbations. It can be proved rigorously for $k=0$. For $\Lambda_{2}>\Lambda_{2 m i n}$ the sign of the derivative changes and therefore we should expect a stable soliton branch. However, the VK criterion cannot be applied to this case. It is connected with very fine details in the proof for the VK-type criteria (for details see [11]). For the NLS case the proof is based on use of the oscillation theorem for scalar Schrodinger operators, which appear after linearization of the NLS on the background of the soliton. However, for the linear stability problem for the FF-SH solitons, instead of scalar operators, we have a matrix $(2 \times 2)$ Schrodinger operators for which the oscillation theorem does not hold ${ }^{2}$. Therefore the VK-type criterion, being a sufficient criterion for instability, cannot be used for stability. Thus we can expect instability for the region of the negative derivative of $N\left(\Lambda_{2}^{2}\right)$. In the region of positive derivative, nevertheless, one can make a conclusion about stability by using a combination of the (incomplete) VK criterion and the Lyapunov approach (this analysis will be published soon [17]). As first shown in [13] (for details see also [11]) the Hamiltonian of the FF-SH system is bounded from below for a fixed Manley-Rowe integral. A key point for stability is to consider the dependence $H(N, P)$ for soliton solutions. If this dependence is monotonic and unique then the soliton solution will be stable. Indeed, the boundedness of the Hamiltonian from below means that solutions realizing its minimum will be stable in accordance with the Lyapunov theorem. In the case of a unique surface $H(N, P)$ this minimizer will belong to this surface. Strictly speaking, the latter needs also compactness of the considered functionals, which can be proved by standard methods for such systems. If the function $H(N, P)$ is not monotonic, then there exist several branches for fixed $N$ and $P$, i.e. $H=H(N, P)$ represents a set of separate surfaces. Then the solitons from the lower branch, or from the lower surface, will be stable only in the Lyapunov sense.

As for the stability of solitons near a supercritical bifurcation, this problem has some peculiarities. As shown before the soliton family near the boundary $\Lambda_{1}=0$ transforms into NLS solitons, generally speaking, independently of the problem dimension. In this case the most dangerous perturbations will be disturbances of the modulation type. Their dynamics will be described by the time-dependent nonlinear Schrodinger equation. It is well known (see, e.f., [12]) that only one-dimensional solitons are stable with respect to perturbations with the same dimension. For instance, the solutions (23) will be stable with respect to one-dimensional modulation perturbations, guaranteed, in particular, by the criterion (2). Thus, in the one-dimensional case the soliton boundary in the parameter space coincides with the stability boundary. However, for dimensions $D \geq 2$ the NLS solitons are known to be unstable. ¿From another perspective, the boundedness of

\footnotetext{
${ }^{1}$ We note that such a tendency was also observed numerically in [16] for the FF-SH solitons in one particular case, but the authors did not give any explanation of this fact.

${ }^{2}$ Recall that the oscillation theorem for the scalar Schrodinger operator establishes a correspondence between a number of nodes of the wave function and a level number.
} 
the Hamiltonian for fixed $N$ provides stability of solitons realizing the minimum of $H$. This means that for $D \geq 2$, in the parameter space the stability region is separated from the soliton boundary.

\section{CONCLUDING REMARKS}

It is necessary to emphasize that the results obtained in this paper about the behavior of solitons near both the supercritical and subcritical bifurcations are in complete agreement with a general theory given in $[6,9]$. It is interesting to note that if the phase mismatch parameter $\Omega$ is negative for stationary solitons $(v=0)$ only the supercritical bifurcation is possible; the subcritical bifurcation is forbidden. For propagating solitons we have another possibility. For instance if

$$
\left(1-\frac{\kappa}{2}\right) v^{2}+\Omega<0
$$

which can be satisfied, for instance, if

$$
\kappa<0, \text { or } p_{2}>2 p_{1}
$$

and then necessarily $\Omega<0$, the only possible bifurcation is the subcritical one. Note, that equality in (40) recovers the Galilean invariance of the FF-SH system. Thus, the soliton family significantly depends on the phase mismatch $\Omega$ and $\kappa$ (i.e. $p_{2}-2 p_{1}$ ) with a large asymmetry with respect to these parameters. The latter will be interesting to observe in experimentally, for instance, in nonlinear optics.

We would like to underline once more that the results of this paper can be easily extended to the multi-dimensional case. In particular, the main conclusion about the character of solitons near subcritical and supercritical bifurcations holds in the multi-dimensional case. For a dimension $D \geq 2$ solitons near a supercritical bifurcation for the FF amplitude will coincide with $D$-dimensional NLS solitons, and the SH amplitude will be proportional to square of the FF amplitude as it is in 1D case. When approaching a subcritical bifurcation point in the multi-dimensional case, the Manley-Rowe integral diverges which results in instability of solitons.

\section{ACKNOWLEDGMENT}

One of the authors (E.K.) is thankful to the Department of Mathematics and Statistics of the Monash University (Melbourne), where this paper was started, and the Nice Observatory, where the paper was completed within the Landau-CNRS agreement, for their hospitality. The work of E.K. was supported by the Russian Foundation of Basic Research under grant no. 00-01-00929 and by the Program of Support of the Leading Scientific Schools of Russia (grant 00-15-96007). The work of E.S. was supported by the Russian Foundation of Basic Research.

[1] Yu. N. Karamzin and A. P. Sukhorukov, Sov. Phys. JETP, 41, 414 (1976); JETP Lett., 20, 339 (1974).

[2] N. Bloembergen, Nonlinear Optics, (Benjamin Inc., N.Y., Amsterdam, 1965).

[3] M. Segev and G. Stegeman, Phys. Today 51, No. 8, 42 (1998); G. Stegeman and M. Segev, Science 286, 1518 (1998); Yu. S. Kivshar, Opt. Quantum Electron. 30, 571 (1998).

[4] V. E. Zakharov and S. V. Manakov, Pis'ma ZhETF (JETP Letters), 18, 413 (1973) (in Russian). 
[5] V. E. Zakharov, S. V. Manakov, S. P. Novikov and L. P. Pitaevsky, Theory of Solitons, (Consultants Bureau, New York, 1984).

[6] V. E. Zakharov and E.A .Kuznetsov , JETP, 86, 1035-1046 (1998).

[7] M. S. Loguet-Higgins, J. Fluid Mech. 200, 951 (1989).

[8] G. Iooss and K. Kirchgassner, C. R. Acad. Sci. Paris 311 , I, 265 (1991).

[9] E. A. Kuznetsov, JETP, 89, 163-172 (1999).

[10] M. G. Vakhitov and A. A. Kolokolov, Radiofizika, 16, 1020 (1973) [Radiophys. Quantum Electron., 16, $783(1973)]$.

[11] E. A. Kuznetsov, in: Optical solitons: theoretical challenges and industrial perspectives, Editions de Physique Springer, V.E. Zakharov and S.Wabnitz (eds.), pp 31-50 (1999).

[12] E. A. Kuznetsov, A.M. Rubenchik and V. E. Zakharov, Phys. Rep. 142, 103 (1986).

[13] A.A.Kanashev and A.M.Rubenchik, Physica 4D, 122 (1981).

[14] L.Berge, V.K.Mezentsev, J.J.Rasmussen, and J.Willer, Phys.Rev.A, 52, R28 (1995).

[15] Yu. S. Kivshar, in: Advanced Photonics with Second-order Optically Nonlinear Processes, A.D.Boardman et al. (eds.), Kluver, Netherlands, pp. 451-475 (1999).

[16] A. V. Buryak and Yu. S. Kivshar, Phys. Lett. A, 197, 407 (1995).

[17] E.A. Kuznetsov, J. J. Rasmussen and E.G. Shapiro, (in preparation). 


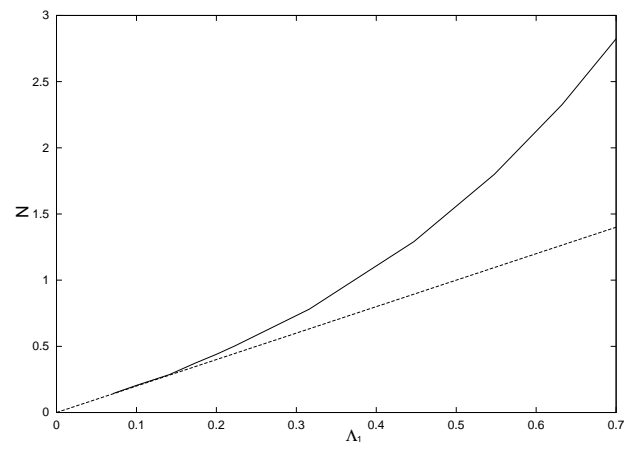

FIG. 1. The dependence of $N$ on $\Lambda_{1}$ for the case $k=0$ and $\Lambda_{2}^{2}=1$ (near the supercritical bifurcation boundary). The straight (dashed) line corresponds to the analytical result (24).

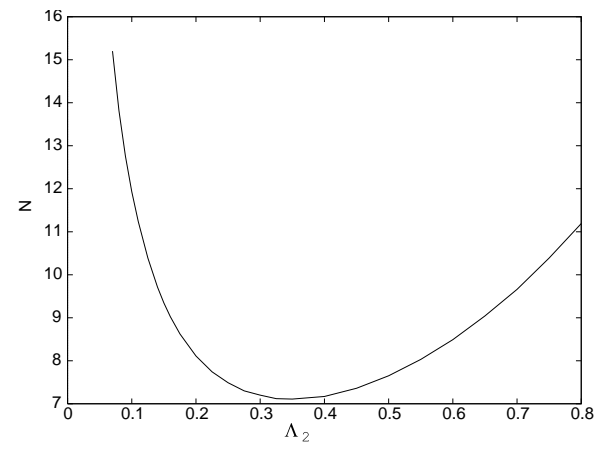

FIG. 2. The dependence $N$ on $\Lambda_{2}$ for the case $\Lambda_{1}^{2}=\Lambda_{2}^{2}+1$ and $k=1 . \Lambda_{2}=0$ corresponds to the subritical bifurcation point. 


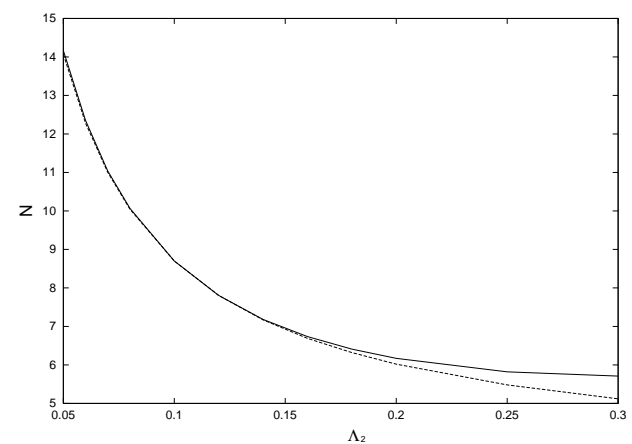

FIG. 3. The curve $N\left(\Lambda_{2}\right)$ for $\Lambda_{1}^{2}=\Lambda_{2}^{2}+1$ and $k=0$ near the subcritical bifurcation boundary $\left(\Lambda_{2}=0\right)$; the dashed line displays the approximation $f\left(\Lambda_{2}\right)$ with $a=0.537$ and $b=3.333$.

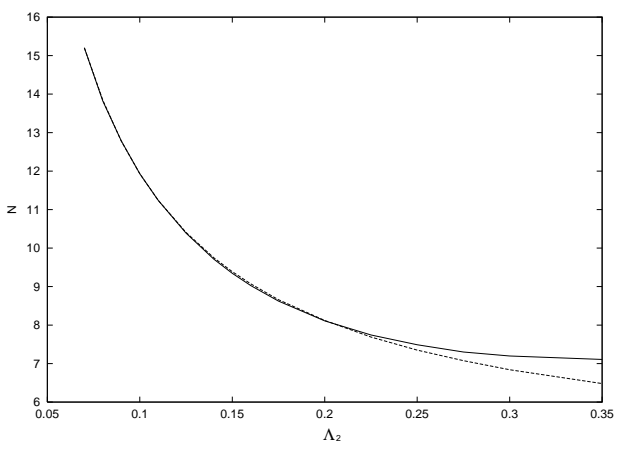

FIG. 4. The curve $N\left(\Lambda_{2}\right)$ for $\Lambda_{1}=1$ and $k=1$ near the subcritical bifurcation boundary $\left(\Lambda_{2}=0\right)$; the dashed line corresponds to the approximation $f\left(\Lambda_{2}\right)$ with $a=0.7630$ and $b=4.299$. 


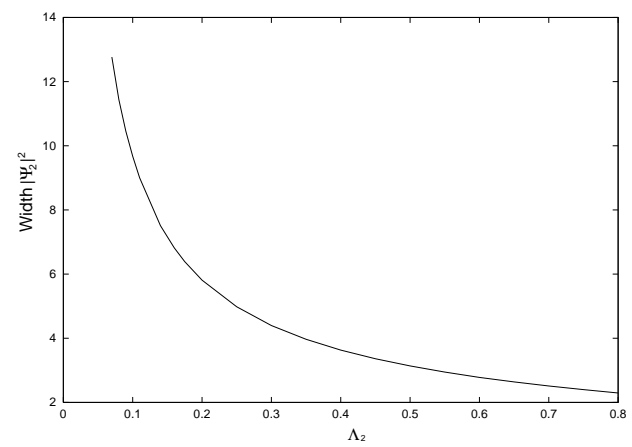

FIG. 5. The maximum value of $\left|\psi_{2}\right|$ near the subcritical bifurcation boundary versus $\Lambda_{2}\left(\Lambda_{1}^{2}=\Lambda_{2}^{2}+1\right.$ and $k=1$ ).

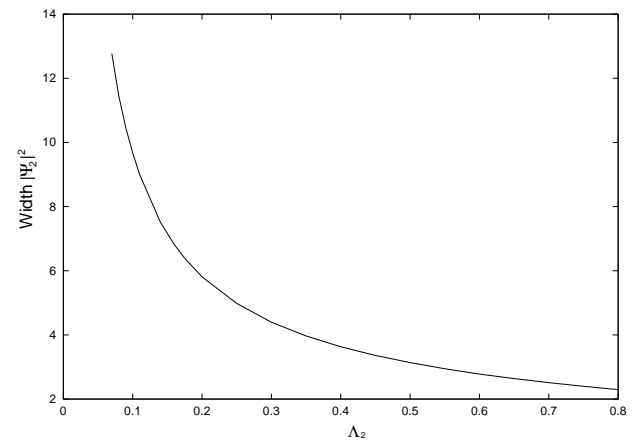

FIG. 6. The width of $\left|\psi_{2}\right|^{2}$ near the subcritical bifurcation boundary versus $\Lambda_{2}\left(\Lambda_{1}^{2}=\Lambda_{2}^{2}+1\right.$ and $\left.k=1\right)$. 

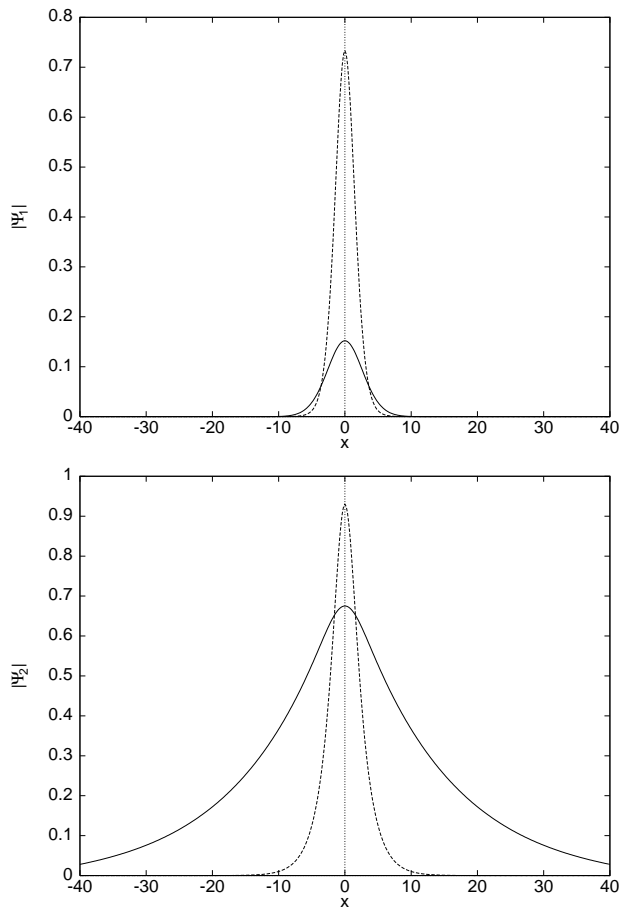

FIG. 7. The functions $\left|\psi_{1}(x)\right|$ and $\left|\psi_{2}(x)\right|$ near the subcritical bifurcation boundary $\left(\Lambda_{1}^{2}=\Lambda_{2}^{2}+1\right.$ and $k=1$ ). Solid line is $\Lambda_{2}=0.1$ and the dashed line $\Lambda_{2}=0.5$. 